\title{
The Experimental Programme
}

\author{
R. Turlay \\ (Département de Physique des Particules Elémentaires, Centre d'Etudes Nucléaires de Saclay)
}

Chairman of the LEP Experiments Committee

Progress in elementary particle physics is the result of a permanent dialogue between theory and experiment. Sometimes a long silence intervenes when new ideas or experimental discoveries are slow to come; in contrast, sometimes the entire process is swept along, creating an extraordinary excitement and blossoming of ideas.

It seems to be in the second of these states that we have been living since the 1970s.

The great idea advanced by Glashow, Weinberg and Salam was to unify the electromagnetic and weak forces through the concept of a spontaneously broken symmetry in a gauge theory. That responded to one of the main concerns of all theoreticians: to have a renormalisable theory and unify all forces, keeping in mind the model of the electromagnetic interaction (QED). A renormalisable theory is one in which amplitudes can be calculated with arbitrary precision in terms of a finite number of experimentally measurable quantities. The best example in QED is the anomalous magnetic moment of the muon:

experiment, $\alpha_{\mu}=1165924 \pm 8.5 \times 10^{-9}$; theoretical prevision [1],

$1165921 \pm 8.3 \times 10^{-9}$

The idea of a spontaneously broken gauge theory is a more subtle concept and the problem of generating masses in the gauge theory has required several steps, some of which were technically difficult. The result is the unification of the two forces with three massive intermediate vector bosons and the photon as the gauge particles.

In parallel, the notion of the quark had been proposed by Gell-man and the quantum theory of strong interactions
(QCD) developed on the same bases of a local symmetry principle and gauge invariance. The vector gauge boson in that case was the gluon.

The two theories, QCD and the electroweak theory, represent our present understanding of particles and forces, and form the so-called "Standard Model".

Many of these theoretical ideas have received marvellous experimental confirmations: neutral currents in 1973, the bound state of the charm quark in 1974 , and the intermediate vector bosons $W^{+}, W^{-}, Z^{\circ}$ in 1983.

\section{Present Status of Standard Model}

What is the present status of the standard model?

The standard theory requires the existence of three lepton families $\left(v_{e}, e\right)$ $\left(v_{\mu}, \mu\right)\left(v_{\tau}, \tau\right)$ and three quark families

\section{LEP Start-up}

With the operation of LEP now imminent, an $\mathrm{e}^{+} \mathrm{e}^{-}$storage ring capable of the highest energy so far achieved in electron positron collisions will be added to the existing accelerator complex of the European Laboratory for Particle Physics, CERN. This machine marks the beginning of a new era in the history of CERN. The laboratory has a tradition of providing and using hadron beams for investigating interactions between elementary particles. It has played a leading role in this field of physics since the first particle beams became available more than 30 years ago. CERN has also a tradition of building and studying physics with storage rings. The ISR was the first large scale hadron collider and the SPS collider is the first step towards the next generation accelerators to be used in particle physics.

The observation in 1983 of the intermediate vector bosons, $W$ and $Z^{\circ}$, at the SPS proton-antiproton collider is currently the most convincing test of present ideas on the electroweak interactions of elementary particles. The new LEP machine will now permit physicists to study details of the decays and the coupling properties of intermediate vector bosons with a hitherto unobtained precision. At centre of mass energies around the $\mathrm{Z}^{\circ}$ mass, $\mathrm{e}^{+} \mathrm{e}^{-}$collisions are a clean laboratory for investigating the properties of the $\mathrm{Z}^{\circ}$, while at total energies above $165 \mathrm{GeV}$, the properties of the $W$ can be studied. Thus the LEP machine is in line with the scientific obligation of the laboratory to study further those particles which were first seen at CERN and it exploits the available technical know-how on building and operating storage rings.

An indication that LEP meets the present needs and that it is widely accepted by the physics community can be seen from the great interest of particle physicists in participating in LEP experiments.
The four interaction regions are occupied by four detectors, which are operated by teams of 300 to 500 scientists from 26 countries.

The LEP machine and its experiments have put a heavy strain on the budgets of all laboratories involved, in particular those in Europe, but the common effort has made this technical adventure into a new domain of physics possible. During the coming years, all those who have endeavoured to get the accelerator and the experiments ready will be rewarded with exciting physics from an excellently designed $\mathrm{e}^{+} \mathrm{e}^{-}$collider. For CERN, LEP is more than a guarantee of high quality physics up to the end of the century; the investment in LEP will help to secure the future of the laboratory beyond the year 2000 .

\section{W. Bartel \\ (Chairman, EPS High Energy and Particle Physics Division)}




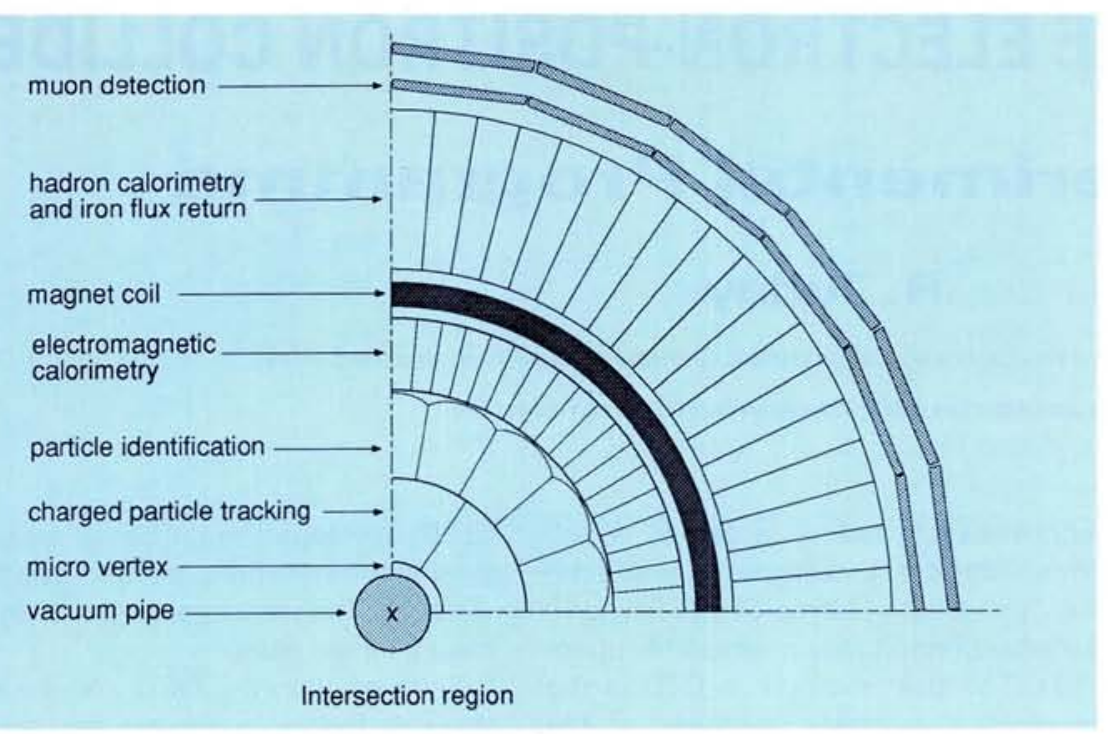

Fig. 1 -Cross-section of an idealized LEP detector.

(u, d) (c, s) (t, b). The forces are mediated by the four electroweak gauge bosons $\mathrm{W}^{+}, \mathrm{W}^{-}, \mathrm{Z}^{\circ}$ and $\gamma$, and eight coloured gluons for the strong force.

In this catalogue only the $t$ quark is missing today; its mass should be larger than the present experimental limit of 40 $\mathrm{GeV}$. The existence of three families is not specific to the Standard Model, nor is it restrictive. So, one of the open questions is "are there more than three families?"

In presenting a particle catalogue one should introduce the scalar Higgs particle. Intimately connected with spontaneously broken symmetry, the "Higgs mechanism" [2] is an ingenious theoretical trick which gives mass to the vector bosons necessary in the theory. The simplest scenario postulates the existence of one scalar neutral particle $\mathrm{H}^{\circ}$. The hunting of this particle is one of the main aspects of all the current experimental programmes.

Electroweak processes can be described using three parameters: the gauge coupling constants $g_{1}$ and $g_{2}$ and the Higgs-field vacuum expectation value $v$. Any other three quantities can be chosen with the only requirement that they obey the theoretical relations of the theory. The following parameters are taken in direct connection with experiments:

- the fine structure constant, $\alpha=1 / 137.0359895(61)$ from $a_{\mu^{\prime}}$

- the Fermi constant, $G_{\mu}=1.166344(11)$ from $\mu$ lifetime, - the mixing angle, $\sin ^{2} \theta_{\mathrm{W}}=0.231$ \pm 0.006 obtained from neutrinoquark scattering.

One sees how the last "new" parameter of the Standard Model is poorly known compared to the coupling constants $\alpha$ and $G_{\mu}$, although its degree of the $\mathrm{e}^{+} \mathrm{e}^{-}$collider with enough energy to produce $\mathrm{Z}^{\circ} \mathrm{s}$ and $\mathrm{Ws}$ is obviously such a tool. This explains the 1980 decision to build the LEP machine (see description by $E$. Picasso and G. Plass in this issue [3]). The goal of the LEP project has always been an $\mathrm{e}^{+} \mathrm{e}^{-}$collider with an energy of $100 \mathrm{GeV}$ per beam: whereas about $46 \mathrm{GeV}$ per beam is needed to produce single $Z^{\circ} \mathrm{s}, 82 \mathrm{GeV}$ per beam is needed to produce $\mathrm{W}^{+} \mathrm{W}^{-}$pairs. Financial reasons motivated the decision in 1981 to introduce a first step in the programme at $50 \mathrm{GeV}$ per beam, called LEP phase I. This is the energy to be given to the experimental teams this summer, and it is this experimental programme which we shall discuss [4].

\section{LEP PHASE I EXPERIMENTAL PROGRAMME}

precision is the result of 10 years of hard experimental work. Testing the theory at its quantum level (as well as QED) will be possible if the very precise predictions beyond the lowest order, the so-called "electroweak radiative corrections" calculated in the renormalisable theory can be compared with equivalently good experimental results. Such an experimental verification will establish the Standard Model as a successfully quantized field theory.

In the lowest order framework, the Weinberg angle parameter $\sin ^{2} \theta_{W}$ may be defined in different but equivalent ways. This equivalence disappears in higher orders where one hopes really to test the Standard Model. At the present time the experimental errors are insufficient, and it is LEP which will allow high precision tests of the electroweak radiative corrections via measurements of $M_{\mathrm{z}}, M_{\mathrm{w}}$ and $\sin ^{2} \theta_{\mathrm{w}}$. Knowledge of any pair of these three quantities enables a test of higher order corrections.

So the Standard Model offers physicists a solid base for understanding particle physics. Pushing it to its ultimate limit requires a "specialized laboratory";

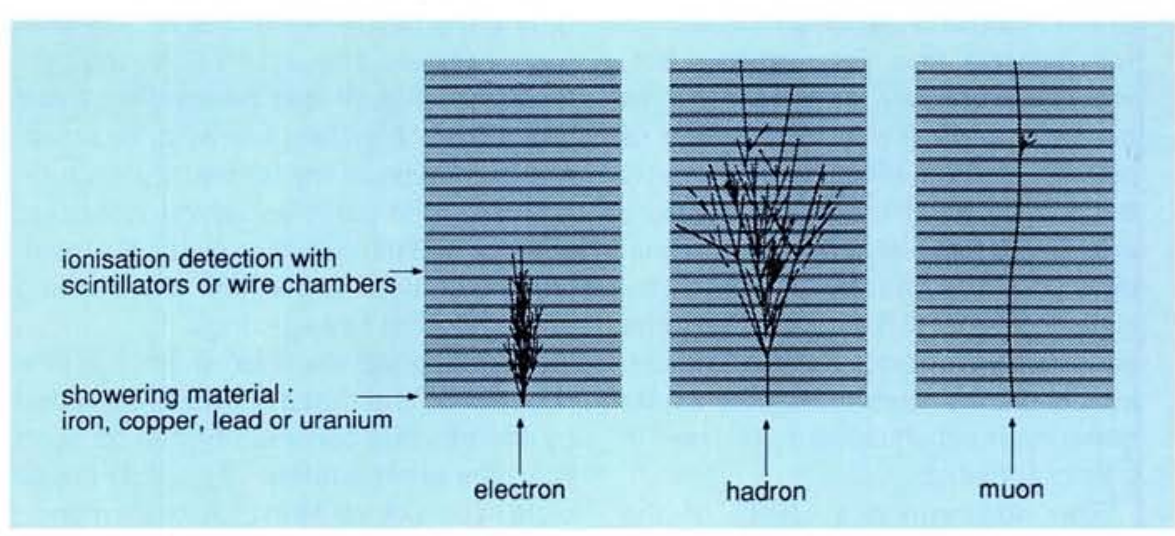

Fig. 2 - Schematic behaviour of an electron, a hadron and a muon in a calorimeter. 


\section{$Z^{\circ}$ Width and Mass}

A scan in energy across the $Z^{\circ}$ resonance peak will provide two independent experimental numbers: the mass and width of the $Z^{\circ}$. Typical precisions expected are:

$$
\begin{aligned}
& \Delta M_{\mathrm{z}} \cong 20 \mathrm{MeV} \\
& \Delta \Gamma_{\mathrm{z}} \cong 50 \mathrm{MeV}
\end{aligned}
$$

The main corrections to the BreitWigner line shape of the $Z^{\circ}$ are the QED radiative corrections. These will alter the line shape, shifting the $Z^{\circ}$ peak and giving rise to a long radiative tail for energies above $M_{z}$. QCD corrections in the final state will also contribute to systematic errors.

\section{Width of the $Z^{\circ}$, number of lepton fami-} lies

The width of the total decay of the $Z^{\circ}$ into fermion-antifermion pairs expressed in terms of partial widths is given as:

$$
\begin{aligned}
& \Gamma_{Z^{\circ}-\text { all }}=0.17 N_{v}+0.086 N_{\ell \pm} \\
& +0.38 N_{-1 / 3}+0.30 N_{2 / 3} \mathrm{GeV}
\end{aligned}
$$

With theoretical errors due to $\rightarrow$

$$
\begin{aligned}
& =\left|\begin{array}{c}
2645 \pm 20 \pm 30 \pm 10 \pm 4 \\
! \\
!
\end{array}\right| \mathrm{MeV} \\
& \alpha_{\mathrm{s}} m_{\mathrm{t}} \sin ^{2} \theta_{\mathrm{w}} \mathrm{Z}^{\circ} \text { mass }
\end{aligned}
$$

Following this formula we see that any extra neutrino generation beyond the three known generations will give a 170 $\mathrm{MeV}$ additional contribution to the width, well beyond the theoretical and experimental errors evaluated today.

The LEP experimental results should be able to reveal the existence of any additional light neutrino or for that matter, any light neutral object which weakly couples to the $Z^{\circ}$.

Other experimental methods can be used to count the number of neutrino types (known as neutrino counting in the current jargon):

- At the $Z^{\circ}$ peak, the absolute value of the cross-section $\mathrm{e}^{+} \mathrm{e}^{-} \rightarrow \mu^{+} \mu^{-}$can be calculated. Then assuming that the width $\Gamma_{\mu \mu}$ is known from the electroweak model $\Gamma_{\text {tot }}$ can be obtained. Here the experimental difficulty lies in obtaining the absolute value of the luminosity. - The study of the process $\mathrm{e}^{+} \mathrm{e}^{-}-\gamma+$ nothing. In the neighbourhood of the $Z^{\circ}$ peak, the size of this cross-section depends upon the partial width of $Z^{\circ} \rightarrow$ neutral species. The differential crosssection versus the photon energy shows a peak in the photon energy, whose height is proportional to the number of neutrino types. The absolute value of this peak must be separated from the background from $\mathrm{e}^{+} \mathrm{e}^{-} \rightarrow \mathrm{e}^{+} \mathrm{e}^{-} \gamma$ where both $\mathrm{e}^{+}$and $\mathrm{e}^{-}$are lost in the beam pipe or in the cracks of the detector and are not recorded.
Mass of the $Z^{\circ}$, contribution to radiative corrections

The present error on $M_{z}$ is $\pm 1.5 \mathrm{GeV}$. The error on the $Z^{\circ}$ mass at LEP will be $\cong 50 \mathrm{MeV}$. This big improvement will remove almost all theoretical errors due to $M_{z}$ in the radiative corrections. (Within the radiative corrections that total about $7 \%$, the new theoretical uncertainty due to the mass will be of $0.1 \%$.) We should add that determining the $W$ mass with equal precision will give a consistency check on the calculation of radiative corrections, but this measurement will only be possible at LEP 200.

\section{Asymmetry in the Standard Model: Value of $\sin ^{2} \theta_{w}$}

The largest contribution to the error on radiative corrections will then come from $\sin ^{2} \theta_{w}$. From the forward-backward asymmetry in the process $\mathrm{e}^{+} \mathrm{e}^{-}=$ $\mu^{+} \mu^{-}$its value can be extracted, but the asymmetry is small and the process is delicate. To obtain a reasonable error in the determination it will be necessary to have long runs of almost 100 days at a luminosity of $10^{31} \mathrm{~cm}^{-2} \mathrm{sec}^{-1}$. We then have to face up to making careful measurements of the records and this may require many years.

Asymmetry measurements using left or right polarized fermions $\left(\mathrm{e}^{+} \mathrm{e}^{-}=\right.$ $\tau^{+} \tau^{-}$) are more sensitive to the Weinberg angle and may be chosen instead of the forward-backward asymmetry. The goal is to measure $\sin ^{2} \theta_{W}$ with a precision of 0.0004 .

\section{Neutral Higgs Searches}

The mass of the neutral Higgs is not known in the Standard Model and Higgs searches are carried out each time a new energy range is available. From theory, we know an important feature of the Higgs: its couplings to fermions and bosons are proportional to their masses. This guides considerations of $\mathrm{H}^{\circ}$ production and decay (see page 94).

All in all, LEP phase I detectors should be able to discover the neutral Higgs if its mass is $\leq 40 \mathrm{GeV}$.

\section{New Particles}

The Standard Model has some limitations, and thus theories "beyond the Standard Model" are being intensively studied. All of them lead to new particles: supersymmetric partners of all known particles, additional heavy bosons, heavy leptons, heavy quarks, preons... It is clear that the discovery of any of these will be of great importance and will lead to a new physics programme and surely to proposals for a new machine.

\section{THE DETECTORS FOR LEP}

We have described a plausible physics programme for the first phase of LEP. Collisions will take place at a rate of about 1 per second leading to the production on average of 40 particles per event. Physicists would like to detect, measure and identify all particles produced in those collisions.

If one does not want to miss particles, the detector must be hermetically closed, thus covering the full $4 \pi$ steradians. Whereas the ideal detector, like the one sketched in Fig. 1, would be spherical, this is technically not easy and the usual form chosen is that of a cylindrical barrel, closed at each end by a cap.

Charged particles must be detected before they interact in a serious way with the material of the detector. This implies that detector elements immediately outside the beam pipe will have to be as "transparent" as possible. Such elements are the ones used to localize precisely the position and trajectory of charged particles. Beyond these layers come "destructive" detectors such as calorimeters, which transform the initial particle state into a detectable ionization process.

We have sketched in Fig. 2 the behaviour of an electron, a hadron and a muon entering a typical calorimeter. It is clear from this figure that the identification of electrons must take place first, after traversing the smallest amount of material, and that the hadron and muon detectors can be placed in the outer layers of the apparatus.

The identification of particles is essential to understanding the underlying physics. Leptons for example, at $\mathrm{e}^{+} \mathrm{e}^{-}$ colliders are often produced as pairs, but leptons can also be the stamp of an unknown "exotic" particle or more commonly the decay of a charm or beauty state. In the latter case the decayed lepton will often have to be found inside a jet of particles. $A$ jet is the name given to a collection of particles coming from the hadronisation of an initial quark or a gluon. Identification of individual particles within a jet means that the cells of detection in the calorimeter will have to be as small as possible. Hence the importance of detector granularity.

Although these necessary ingredients are common to all the experiments, the techniques used and the particular emphasis given by each Collaboration differs, leading to four very powerful detectors: ALEPH, DELPHI, L3 and OPAL.

On a very general basis, the three experiments ALEPH, OPAL and DELPHI are similar in concept, except that DELPHI 
has an important "plus", the RICH detector, which will make it the only experiment able to identify $\pi, p$ and $K$ up to an energy of $20 \mathrm{GeV}$. In contrast, L3 has followed a very original concept and has fixed the goal of "measuring photons, electrons and muons with a resolution of $\Delta p / p \cong 1 \%$ ".

Instead of describing sequentially each of the 4 detectors, I shall start from the "ideal" detector sketched in Fig. 1. I shall cite the name of the instrument and give the particular specificity for each of the Collaborations' designs and, for simplicity, I shall refer to the barrel description only. Details of all the four detectors can be found in their proposals [5].

\section{Tracking Device}

To determine a particle's momentum we need to measure its trajectory in a known magnetic field. The local positions in space of the ionizing particle are found either by measuring the coordinates of the ionization compared to those of wires very precisely located in space (drift chambers, multiwire proportional chambers, Time Expansion Chamber (TEC)) or by measuring the time taken by the track of ionized electrons to drift to a plane of wires and pads (Time Projection Chamber (TPC)). Generally the tracking is done in several different steps:

a) The vertex detector: just outside the beam pipe is a very precise wire chamber which detects and localizes the charged tracks as soon as they escape from the collision region. In OPAL, the diameter $\varnothing=235 \mathrm{~mm}$, length $=1 \mathrm{~m}$, the spatial resolution $\sigma=50 \mu \mathrm{m}$, whereas L3 uses the inner ring of the TEC: $\varnothing=180 \mathrm{~mm}$, length $=1 \mathrm{~m}, \sigma=$ $45 \mu \mathrm{m}$. Two experiments, ALEPH and DELPHI, have undertaken the construction of "micro"-vertex detectors using silicon strips with much better precision, $\sigma=5 \mu \mathrm{m}$. The device will be used to find the decay vertices of short lived particles with proper decay path lengths of 30 to $300 \mu \mathrm{m}$.

b) Inner tracking chamber: (ALEPH and DELPHI) wire chambers used primarily for triggering purposes do not need to be so precise $(100 \mu \mathrm{m})$.

c) Large tracking chamber: designed primarily to measure momentum, here the particle trajectory can develop over distances of one meter or more. A TPC was chosen for ALEPH $(\varnothing=3.6 \mathrm{~m}$, length $=4.8 \mathrm{~m}, \sigma=150 \mu \mathrm{m})$ and DELPHI $\varnothing=1 \mathrm{~m}$, length $=2.8 \mathrm{~m}, \sigma=150$ $\mu \mathrm{m})$. OPAL uses the large volume $(\varnothing=$ $3.8 \mathrm{~m}$, length $=4.5 \mathrm{~m}, \sigma=230 \mu \mathrm{m}$ ) of a wire jet chamber. In L3 the diameter is smaller in the TEC $(\varnothing=0.9 \mathrm{~m})$ but the precision better, $\sigma=45 \mu \mathrm{m}$.

\section{Particle Identification}

Identification of particular species of hadrons, $\pi, \mathrm{p}$ or $\mathrm{K}$ is also desirable but that cannot be done with a calorimeter. One can however use information from the tracking chamber on the ionization energy loss per unit of track length $d E / d x$, or use a more sophisticated Ring Imaging Cherenkov Detector ( $\mathrm{RICH}$ ) in which case the individual particle's Cherenkov light cones are differentiated.

In this respect, DELPHI is unique among the four experiments. $\mathrm{RICH}$ data supplement the $d E / d x$ information and provide this experiment with $\mathrm{e}, \pi, \mathrm{K}$ and p identification from 0.3 to $25 \mathrm{GeV}$ with good efficiency. The operating principle of the RICH is simple: Cherenkov light produced in a cone whose angle is proportional to the particle's velocity is imaged as a ring and measured in a sys tem of drift tubes. The conversion of photons is done within the gas of the drift tubes and the electrons created drift along the tubes in the direction of the magnetic field (the same principle as the TPC or HPC). The electrons reach an anode and cathode strip which determines their coordinates, i.e. the diameter of the circle of the light cone image. A very clever combination of liquid freon, cell and freon gas volume give a good separation of particles.

The $d E / d x$ information can be obtained in all the detectors from either the TPC or wire chambers. The result is a separation between $\mathrm{e}, \pi, \mathrm{K}$ and $\mathrm{p}$ up to $\cong$ $8 \mathrm{GeV} / \mathrm{c}$ for $\mathrm{e}$ and $\pi$ and less for $\pi$ and $\mathrm{K}$.

The construction of the $\mathrm{RICH}$ counters within a small volume has been a difficult challenge for the DELPHI Collaboration, but they are now operational and have proved successful in tests.

\section{Electromagnetic Calorimeter}

A calorimeter in elementary particle physics is a volume in which a particle loses all its energy by multiple electromagnetic or strong interactions. The ionization created by the secondary particles corresponding to this loss is registered. This ionization could be detected as the light in a scintillator or Cherenkov light in lead glass, or as electronic signals in liquid argon or wire chambers. The use of these calorimeters is twofold: energy measurement and particle identification, primarily electrons and photons compared with hadrons.

Typically, incident electrons and photons are converted into low energy electrons and photons in materials such as lead. A full shower develops which contains all of the incident energy in a depth of about 20 radiation lengths. (The radiation length in lead is $0.56 \mathrm{~cm}$.) Thus the electromagnetic calorimeters have a small dimension in depth depending upon the choice of showering material and detection technique.

The electrons are detected in either a gas filled proportional wire tube and pulses summed in geometrical towers (ALEPH), or in a gas sampled volume and drifted to an end plate (DELPHI High density Projection Chamber, HPC). In transparent materials such as lead glass blocks (OPAL 9440 blocks of $10 \times 10$ $\mathrm{cm}^{2}$ ) or Bismuth Germanium Oxide crystals (BGO) in L3 $(7680$ crystals of $2 \times 2 \mathrm{~cm}^{2}$ ) the Cherenkov light is collected in phototubes. By far the most precise of these detectors is the BGO calorimeter which has many advantages (high resolution, compactness) but which suffered from its very high cost and the difficulties of crystal production in large quantities. The L 3 Collaboration overcame these difficulties and all the crystals of the barrel have been tested and installed.

The Table gives the performances of electromagnetic detectors of the four Collaborations, to which should be added the following:

- To improve the spatial and energy resolution for isolated electrons, OPAL constructed a presampling detector between the coil assembly and the lead glass array. The resolutions which are given include the presampler's improvement.

- The energy resolutions given in the Table are the results of measurements in test beams. The resolution in situ will not be quite as good for some of the detectors because of the amount of material in front of them. In addition the knowledge of the calibration and its monitoring for these detectors at a level of $1 \%$ is a challenge and remains to be proven.

\section{The Magnets}

Concerning the solenoidal magnet designs: to be safe, OPAL was required to built a warm "classical" coil, since, at the time of the decision, only a few small superconducting coils were built. We can say today that the extrapolation of the superconducting coils to $5 \mathrm{~m}$ diameter for ALEPH and DELPHI has been a success. With a diameter of $12 \mathrm{~m}$, the L 3 magnet is also quite a success, even using "warm" classical technology.

Because of geometrical limitations all four experiments are equipped with cylindrical coils, the dimensions of which vary with the physics choices of each Collaboration. Because these coils typically have material thickness equivalent to 0.7 to 1.5 radiation lengths, the Colla- 
Performance of the EM Detectors

\begin{tabular}{|l|c|c|}
\hline & $\begin{array}{c}\text { Energy Resolution } \\
\triangle E / E, \% / \sqrt{ } E\end{array}$ & $\begin{array}{c}\text { Spatial resolution } \\
\sigma_{x, y}, \mathrm{~mm}\end{array}$ \\
\hline ALEPH & 18 & 2.5 \\
DELPHI & 17 & $3-10$ \\
L3 & 3 & 3 \\
OPAL & 7 & 3.7 \\
\hline
\end{tabular}

borations had to decide whether to put the electromagnetic calorimeter inside or outside the coil cylinder. OPAL decided to detect electrons and photons outside the coil assembly. In their case a 0.4 T field is produced in a $6.1 \mathrm{~m}$ long, $4 \mathrm{~m}$ diameter warm coil. ALEPH and DELPHI have placed their electromagnetic calorimeters inside superconducting coils of respectively $5 \mathrm{~m}$ diameter at $1.5 \mathrm{~T}$ and $5.25 \mathrm{~m}$ at 1.2 T. These two experiments use TPC chambers and thus require very homogeneous fields $\left(10^{-5}\right)$ for which additional compensation coils were necessary. L3 Collaboration which has focussed on muon measurements with a very high precision, required a large volume. Their choice was a very large warm coil, $12 \mathrm{~m}$ in diameter and $12 \mathrm{~m}$ long, operating at $0.4 \mathrm{~T}$.

\section{Hadron Calorimeter}

Most hadrons after traversing the electromagnetic calorimeter and the magnet coil have not yet begun to shower; more material is needed. The flux-return yokes for the magnetic fields are large iron structures constituting the main support of the four experiments. In ALEPH, DELPHI and OPAL, this iron structure is laminated and has been instrumented with streamer tubes placed between the iron slabs.

This massive structure then serves to convert the interacting hadron into a shower of particles analogous to the electromagnetic calorimeter. The characteristic thickness of each of these hadron calorimeters is one meter of instrumented iron. The resolution in energy is $\Delta E / E=80 \% / \sqrt{ } E$. In the $L 3$ case the iron yoke is not instrumented; instead they chose to surround the BGO detector with a uranium hadron calorimeter. Outside 4 nuclear collision lengths of uranium they added 2 collision lengths of copper to absorb completely the energy of incident hadrons.

\section{Muon Identification}

Muons are identified as charged tracks which fully penetrate all the material of the electromagnetic and hadron calorimeters. A fit connecting a track in the central detector with the external track is required; for this purpose ALEPH, DELPHI and OPAL have inserted tracking detectors into the iron structure and placed double layers of tracking chambers outside the iron. After its identification, the muon momentum measurement is done using the central tracking device. In the L3 detector the muon measurement is done outside the hadron calorimeter in the very large magnetic volume using a three layer system of extremely precisely positioned wire chambers $(40 \mu \mathrm{m})$, separated by $1.5 \mathrm{~m}$. Their goal of a momentum measurement at the precision of $1 \%$ is achieved.

\section{Luminosity Monitor}

Absolute and relative luminosity measurements are needed to normalize the data. In $\mathrm{e}^{+} \mathrm{e}^{-}$machines this is done by measuring the energy and angle of the Bhabha electrons. The angular distribution is strongly peaked at $0^{\circ}$ and one needs very forward detectors to detect them. Because of the beam pipe diameter and the position of the low $\beta$ quadrupoles close to the intersection points, experiments will start to detect Bhabha electrons only above $3^{\circ}$. A tracking chamber associated with an electromagnetic calorimeter gives the precision required for the luminosity measurement.

\section{Electronics}

The number of electronic signals from the different parts of the detector will be very large. We have seen that on average about 10000 read-out channels are used for lead glass or BGO calorimeters, 50000 for a TPC or wire chamber, and the same for a hadron calorimeter. The peak is the 220000 channels of the ALEPH electromagnetic calorimeter! One is speaking about $10^{6}$ analogue signals per event as a realistic assessment. These analogue signals are integrated and multiplexed near the detector modules. The requirements in electronic effort at this first stage has pushed the development of monolithic versions of integrated circuits so as to save space, time ... and money. These define the "first" trigger level which must decide in the shortest time (few hundred nanoseconds) if an interesting event has occured. Then analogue to digital conversion begins. Microprocessors are used as a more sophisticated second level trigger using for instance track reconstruction and correlations between differents part of the detector (e.g. the association of the fit of a track and a corresponding deposit of energy in a cell of the electromagnetic calorimeter). The last step is the read-out into the data acquisition system where Camac, Fastbus or VME systems are used with processor memory and central logic on each crate. They are centralised in the main control rooms. Each experiment at this stage has developed its own data acquisition system which includes large computers and/or processor farms. These data acquisition systems record events on disk and on magnetic tape from which starts the final production of data ready for physics analysis.

One of the main installation problems is the cabling needed for the massive output of electronic information. In each experiment several tens of kilometers of cables have to be carefully placed and fitted into the tight detector geometry. Millions of connexions must be verified, a non-negligible part of the entire game.

\section{Computing}

Having the machine ready on time and the detectors perfectly polished is not enough if the analysis programmes are not ready, and so over the past several years, full simulations of events (also called Monte Carlo events) have been undertaken in parallel to the work of preparing the detectors. Based on large "universal" programs these simulated events need more processing time on big computers than it takes to record real data during the experiment. To produce millions of realistic simulated events, each Collaboration needs the equivalent calculating power of the largest computers currently available.

\section{CONCLUSION}

Over the next two years, a straightforward programme is foreseen and work will be done to exploit the first step in energy of LEP phase I. In parallel, thought and technical developments are being invested now to upgrade the machine to $200 \mathrm{GeV}$ and to consider the possibility of running with longitudinally polarized electron and positron beams. Altogether this is a solid programme which will extend up to the end of the century.

\section{REFERENCES}

[1] Bailey J. et al., Nuclear Physics B 150 (1979) 1-75.

[2] Aguilar-Benitez M. and Herrero M.J., 'The Higgs Connexion' this issue of Europhys. News 20 (1989). 92.

[3] Picasso E. and Plass G., 'The Machine Design' this issue of Europhys. News 20 (1989) 80.

[4] Physics at LEP - Eds. J. Ellis and R. Peccei - CERN 86-02 - Vols. I and II - 21 February 1986.

[5] ALEPH - Technical proposal - CERN/ LEPC/83-2 - May 1983. DELPHI - Technical proposal - CERN/LEPC/83-3 - May 1983. L3 - Technical proposal - CERN/LEPC/83-5 - May 1983. OPAL - Technical proposal CERN/LEPC/83-4 - May 1983. 